\title{
Publisher's Note: Biochemical Machines for the Interconversion of Mutual Information and Work [Phys. Rev. Lett. 118, 028101 (2017)]
}

Thomas McGrath, Nick S. Jones, Pieter Rein ten Wolde, and Thomas E. Ouldridge (Received 13 January 2017; published 27 January 2017)

DOI: 10.1103/PhysRevLett.118.049902

This Letter was published online on 10 January 2017 with an omission of an affiliation. The author affiliations should read as

Thomas McGrath, ${ }^{1}$ Nick S. Jones, ${ }^{1}$ Pieter Rein ten Wolde, ${ }^{2}$ and Thomas E. Ouldridge ${ }^{3, *}$

${ }^{1}$ Department of Mathematics, Imperial College London, London SW7 2AZ, United Kingdom

${ }^{2}$ FOM Institute AMOLF, Science Park 104, 1098 XE Amsterdam, Netherlands

${ }^{3}$ Department of Bioengineering, Imperial College London, London SW7 2AZ, United Kingdom

The affiliations have been corrected as of 12 January 2017. The affiliations are correct in the printed version of the journal. 